\title{
PRIMARY AND COPRIMARY DECOMPOSITIONS
}

\author{
by D. J. MOORE
}

(Received 22nd June 1972)

Let $R$ be an associative, commutative ring with identity, and let $A$ be a (unitary) $R$-module. It is well known that if $A$ is a Noetherian $R$-module then every submodule of $A$ has a primary decomposition in $A$. The object of the present paper is to dualise this result; that is, to show that if $A$ is an Artinian $R$-module then every submodule of $A$ can be expressed as the sum of a finite number of coprimary submodules of $A$.

There are two possible approaches to this problem. Firstly we could tackle the problem directly, attempting to give statements and proofs of results that are, in some sense, duals of the corresponding results from the classical theory. The second alternative is the one we shall adopt. We shall develop a theory of primary decompositions for an object of an abelian category over the ring $R$. (For the definition, see (3).) Using the formal duality of abelian categories, we shall then obtain the theory of coprimary decompositions that we require.

The first three sections of this paper are concerned with establishing existence and uniqueness theorems for primary and coprimary decompositions. In the fourth section we obtain a standard result for Noetherian $R$-modules, together with its dual. The dual result is a generalisation of a result obtained by Matlis in (2) by using the first approach suggested above. The paper concludes with a simple example to illustrate the ideas we have previously introduced.

I would like to thank Professor D. G. Northcott for his help in the preparation of that part of this paper which is contained in my thesis (Sheffield, 1971).

\section{Primary and coprimary subobjects}

Henceforth $R$ will always denote an associative, commutative ring with identity, and $\mathscr{U}$ will denote an abelian category. $\mathscr{C}$ will denote the centre of $\mathscr{U}$, see (1), and $\phi: R \rightarrow \mathscr{C}$ will be a mapping such that $(\mathscr{U}, \phi)$ is an abelian category over $R$, see (3).

If $\mathscr{U}^{*}$ denotes the dual abelian category of $\mathscr{U}$, there is an obvious mapping $\phi^{*}: R \rightarrow \mathscr{C}^{*}$ (the centre of $\mathscr{U}^{*}$ ) such that $\left(\mathscr{U}^{*}, \phi^{*}\right)$ is also an abelian category over $R$. In this situation $\left(\mathscr{U}^{*}, \phi^{*}\right)$ will be called the dual of $(\mathscr{U}, \phi)$.

For $r \in R$ and any $\mathscr{U}$-object $A \phi(r)$ induces a morphism $\phi(r)^{A}: A \rightarrow A$. If $f \in H(A, B)$, where $H(A, B)$ denotes the set of $\mathscr{U}$-morphisms with domain $A$ and codomain $B$, then we set

$$
r f=f \phi(r)^{A}=\phi(r)^{B} f
$$


$H(A, B)$ thus becomes an $R$-module. Also

$$
\begin{gathered}
(r f)^{*}=r f^{*} \\
(r f) g=r(f g)=f(r g),
\end{gathered}
$$

for any $\mathscr{U}$-morphism with codomain $A$, and

$$
r 1_{A}=\phi(r)^{A} \text {. }
$$

We refer to the morphism $r 1_{A}: A \rightarrow A$ as " multiplication of $A$ by $r$ ".

Let $K$ be a subobject of $A$. Then we shall denote the kernel of the composite morphism $A \stackrel{r 1_{A}}{\longrightarrow} A \rightarrow A / K$ by $K:{ }_{A} r$, or by $r^{-1}(K)$ if there is no danger of confusion, and we shall denote the image of the composite map $K \rightarrow A \stackrel{r 1_{A}}{\longrightarrow} A$ by $r K$.

If $\Omega$ is an ideal of $R$, which is finitely generated by $\left\{\omega_{1}, \omega_{2}, \ldots, \omega_{n}\right\}$, and $K$ is a subobject of the $\mathscr{U}$-object $A$, then we shall set

and

$$
\Omega K=\omega_{1} K+\omega_{2} K+\ldots+\omega_{n} K
$$

$$
K:{ }_{A} \Omega=\left(K:{ }_{A} \omega_{1}\right) \cap\left(K:{ }_{A} \omega_{2}\right) \cap \ldots \cap\left(K:{ }_{A} \omega_{n}\right) .
$$

These definitions may be seen to be independent of the particular finite generating set chosen for the ideal $\Omega$.

We shall adopt the convention that the intersection of an empty set of subobjects of an $\mathscr{U}$-object $A$ equals $A$, and that the sum of an empty set of subobjects of $A$ is the null object.

Our first result will give us basic identities needed to interpret results and their duals.

Lemma 1. Let $K$ be a subobject of the $\mathscr{U}$-object $A$ and let $r \in R$. Then

and

$$
\left[A /\left(K:_{A} r\right)\right]^{*}=r(A / K)^{*}
$$

$$
(A / r K)^{*}=(A / K)^{*}:_{A^{*}} r .
$$

Proof. $\quad\left[A /\left(K:{ }_{A} r\right)\right]^{*}=\left[\operatorname{Coim}\left(A \stackrel{r{ }_{A}}{\longrightarrow} A \rightarrow A / K\right)\right]^{*}$

$$
\begin{aligned}
& =\operatorname{Im}\left((A / K)^{*} \rightarrow A^{*} \stackrel{r_{A^{*}}}{\longrightarrow} A^{*}\right) \\
& =r(A / K)^{*} .
\end{aligned}
$$

Also

$$
\begin{aligned}
(A / r K)^{*} & =\left[\operatorname{Coker}\left(K \rightarrow A \stackrel{r 1_{A}}{\longrightarrow} A\right)\right]^{*} \\
& =\operatorname{Ker}\left(A^{*} \stackrel{r 1_{A^{*}}}{\longrightarrow} A^{*} \rightarrow K^{*}\right) \\
& =\operatorname{Ker}\left(A^{*} \stackrel{r_{A^{*}}}{\longrightarrow} A^{*} \rightarrow A^{*} /(A / K)^{*}\right) \\
& =(A / K)^{*}:_{A^{*}} r .
\end{aligned}
$$

Definition. Let $A$ be an $\mathscr{U}$-object, and let $K$ be a proper subobject of $A$. Then $K$ is said to be a primary subobject of $A$ if, for any subobject $N$ of $A$ such 
that $N \leqq K$ and any $r \in R$ such that $r N \leqq K$, there exists a positive integer $n$ such that $r^{n} A \leqq K$.

Definition. The subobject $K$ of the $\mathscr{U}$-object $A$ is said to be a coprimary subobject of $A$ if the subobject $(A / K)^{*}$ of the $\mathscr{U}^{*}$-object $A^{*}$ is primary.

This definition can be expressed in a more convenient form as a consequence of the following result.

Lemma 2. The subobject $K$ of $A$ is coprimary if and only if the following conditions are satisfied:

(a) $K$ is non-null,

(b) for any subobject $N$ of $A$ such that $K \$ N$, and any $r \in R$ such that $K \leqq\left(N:{ }_{A} r\right)$, there exists a positive integer $n$ such that $K \leqq\left(0:{ }_{A} r^{n}\right)$.

Proof. Note firstly that $K$ is null if and only if $(A / K)^{*}$ is not a proper subobject of $A^{*}$.

Assume now that $K$ is a coprimary subobject of $A$ and that $N$ is a subobject of $A$, with $r \in R$, such that $K \leqq\left(N:{ }_{A} r\right)$, while $K \leqq N$. Then $(A / K)^{*}$ is a primary subobject of $A^{*},(A / N)^{*}$ is a subobject of $A^{*}$ such that $(A / N)^{*} \pm(A / K)^{*}$, and $r \in R$ is such that $r(A / N)^{*} \leqq(A / K)^{*}$. Hence there exists a positive integer $n$ such that $r^{n} A^{*} \leqq(A / K)^{*}$. Therefore $K \leqq\left(0:{ }_{A} r^{n}\right)$, as required.

Conversely, suppose $(a)$ and $(b)$ are satisfied. Let $X^{*}$ be a subobject of $A^{*}$, and let $r \in R$, such that $X^{*} \leq(A / K)^{*}$, while $r X^{*} \leqq(A / K)^{*}$. There exists a subobject $N$ of $A$ such that $X^{*}=(A / N)^{*}$. Since $(A / N)^{*}(A / K)^{*}$ it follows that $K \leq N$, while $r(A / N)^{*} \leqq(A / K)^{*}$ implies that $K \leqq\left(N:{ }_{A} r\right)$. By $(b)$, there exists a positive integer $n$ such that $K \leqq\left(0:{ }_{A} r^{n}\right)$, which, by Lemma 1 , implies that $r^{n} A^{*} \leqq(A / K)^{*}$. Thus $(A / K)^{*}$ is a primary subobject of $A^{*}$, so that $K$ is a coprimary subobject of $A$. This completes the proof of the lemma.

Let $K$ be an arbitrary subobject of the $\mathscr{U}$-object $A$. We set

and

$$
\mathscr{P}_{A, K}=\left\{r \in R: r^{n} A \leqq K \text { for some positive integer } n\right\}
$$

$$
\mathscr{P}_{A, K}^{*}=\left\{r \in R: K \leqq\left(0:{ }_{A} r^{n}\right) \text { for some positive integer } n\right\} \text {. }
$$

Since $K \leqq\left(0:{ }_{A} r^{n}\right) \Leftrightarrow r^{n} A^{*} \leqq(A / K)^{*}$ we deduce that $\mathscr{P}_{A, K}^{*}=\mathscr{P}_{A^{*},(A / K)^{*}}$

Lemma 3. Let $K$ be a subobject of $A$. Then $\mathscr{P}_{A, K}$ and $\mathscr{P}_{A, K}^{*}$ are ideals of $R$. Further, if $K$ is a primary (respectively, coprimary) subobject of $A$, then $\mathscr{P}_{A, K}$ (respectively, $\mathscr{P}_{A, K}^{*}$ ) is a prime ideal of $R$.

Proof. We shall leave the proof that $\mathscr{P}_{A, K}$ is an ideal to the reader.

Suppose that $K$ is a primary subobject of $A$. Then $\mathscr{P}_{A, K}$ is a proper ideal of $R$, since $1 \notin \mathscr{P}_{A, K}$. Next assume that $r_{1} r_{2} \in \mathscr{P}_{A, K}$ while $r_{2} \notin \mathscr{P}_{A, K}$. Suppose that $n$ is a positive integer such that $\left(r_{1} r_{2}\right)^{n} A \leqq K$. Then $r_{1}^{n}\left(r_{2}^{n} A\right) \leqq K$. However, $r_{2}^{n} A \pm K$ since $r_{2} \notin \mathscr{P}_{A}, K$. Since $K$ is a primary subobject of $\bar{A}$ it follows that 
there exists a positive integer $m$ for which $\left(r_{1}^{n}\right)^{m} A \leqq K$. Thus $r_{1} \in \mathscr{P}_{A, K}$, so that $\mathscr{P}_{A, K}$ is a prime ideal of $R$.

The results for $\mathscr{P}_{A, K}^{*}$ follow from this and from the result immediately preceding this lemma. This completes the proof.

Let $P$ be a prime ideal of $R$. The subobject $K$ of $A$ is said to be $P$-primary (respectively, $P$-coprimary) if $K$ is a primary (respectively, coprimary) subobject of $A$ and $\mathscr{P}_{A, K}=P$ (respectively, $\mathscr{P}_{A, K}^{*}=P$ ).

The following three results have proofs which are very similar to the corresponding results from commutative algebra (see, for example, (4), pp. 99-100) so we omit these proofs.

Lemma 4. Let $K$ be a proper subobject of $A$, and let $\pi$ be a proper ideal of $R$. Assume also that the following conditions hold:

(a) for any subobject $N$ of $\dot{A}$ and any $r \in R$ such that $r N \leqq K$, either $N \leqq K$ or $r \in \pi$.

(b) $\pi \subseteq \mathscr{P}_{A, \boldsymbol{K}}$.

Then $\pi$ is a prime ideal of $R$ and $K$ is a $\pi$-primary subobject of $A$.

Lemma 5. Let $K_{1}, K_{2}, \ldots, K_{m}$ be a non-empty finite set of P-primary subobjects of $A$. Then $K=K_{1} \cap K_{2} \cap \ldots \cap K_{m}$ is a $P$-primary subobject of $A$.

Lemma 6. Suppose that $K$ and $N$ are subobjects of $A$. Suppose further that $N$ is a $\mathscr{P}_{A, N}$-primary subobject of $A$. Then either $K \cap N=K$ or $K \cap N$ is a $\mathscr{P}_{A, N^{-}}$primary subobject of $K$.

\section{Existence theorems}

Let $A$ be an object of the abelian category $(\mathscr{U}, \phi)$ over the ring $R$, and let $K$ be a subobject of $A$.

Definition. $K$ is said to have a primary decomposition in $A$ if $K$ can be expressed as the intersection of a finite number of primary subobjects of $A$.

Definition. Suppose that $K \equiv N_{1} \cap N_{2} \cap \ldots \cap N_{m}$ is a primary decomposition of $K$ in $A$. We say this is a reduced primary decomposition of $K$ in $A$ if the following further conditions are satisfied:

(a) $\mathscr{P}_{A, N_{i}} \neq \mathscr{P}_{A, N_{j}} \quad(i \neq j)$

(b) no proper subset of $\left\{N_{1}, N_{2}, \ldots, N_{m}\right\}$ has intersection equivalent to $K$.

By virtue of Lemma 5, and by omitting any superfluous terms, we can obtain a reduced primary decomposition of $K$ in $A$ from any given primary decomposition of $K$ in $A$.

Definition. $A$ is said to be a fully structured object of $\mathscr{U}$ if every subobject of $A$ has a primary decomposition in $A$. 
Definition. $A$ is said to be Noetherian if, for every ascending chain

$$
A_{1} \leqq A_{2} \leqq A_{3} \leqq \cdots
$$

of subobjects of $A$, there exists an integer $n$ such that $A_{n} \equiv A_{n+p}$ for $p=0,1,2, \ldots$.

We shall show that every Noetherian $\mathscr{U}$-object is fully structured. In order to achieve this we shall introduce one auxiliary concept.

Definition. We shall say that $K$ is an irreducible subobject of $A$ if $K$ is a proper subobject of $A$ and if, whenever $K \equiv K_{1} \cap K_{2}$, where $K_{1}, K_{2}$ are subobjects of $A$, then either $K \equiv K_{1}$ or $K \equiv K_{2}$.

Lemma 7. Let $A$ be a Noetherian object of $\mathscr{U}$, and $K$ a subobject of $A$. Then $K$ can be expressed as a finite intersection of irreducible subobjects of $A$.

Proof. To obtain a contradiction, we suppose that $K$ cannot be expressed as a finite intersection of irreducible subobjects of $A$. Then $K$ must be a proper subobject of $A$, since $A$ is by convention an empty intersection of subobjects of itself. Also $K$ must be non-irreducible. Therefore there exist $N_{1}, K_{1}$, proper subobjects of $A$, neither of which is equivalent to $K$, such that $K \equiv N_{1} \cap K_{1}$. We may assume that $K_{1}$ cannot be expressed as a finite intersection of irreducible subobjects of $A$. We may repeat this argument to generate inductively an arbitrarily long non-stationary sequence $K \leqq K_{1} \leqq K_{2} \leqq \ldots \leqq K_{p} \leqq K_{p+1} \leqq \ldots$ of subobjects of $A$. Thus $A$ is not a Noetherian $\mathscr{U}$-object, which is the required contradiction.

Lemma 8. Let $A$ be a Noetherian $\mathscr{U}$-object. Then any irreducible subobject of $A$ is primary.

Proof. Let $K$ be a proper subobject of $A$ which is not primary. We shall show that $K$ is not an irreducible subobject of $A$.

Let $N$ be a subobject of $A$, with $r \in R$, such that $N \leqq K, r N \leqq K$, and $r^{n} A \leqq K$ for every positive integer $n$. Consider the ascending sequence

$$
K:{ }_{A} r \leqq K:{ }_{A} r^{2} \leqq \cdots \leqq K:{ }_{A} r^{n} \leqq K:{ }_{A} r^{n+1} \leqq \cdots
$$

of subobjects of $A$. Let $n$ be the smallest positive integer such that

for $p=0,1,2, \ldots$.

$$
K:{ }_{A} r^{n} \equiv K:{ }_{A} r^{n+p}
$$

We consider the subobject $X=\left(K:{ }_{A} r^{n}\right) \cap\left(K+r^{n} A\right)$ of $A$. Obviously $K \leqq X$. From $N \leqq K:{ }_{A} r^{n}$ and $r^{n} A \$ K$ it follows that $K:{ }_{A} r^{n} \not \equiv K$ and $K+r^{n} A \not \equiv K$.

It remains to show only that $X \leqq K$. Since $K \leqq K:{ }_{A} r^{n}$, we may apply the " modular law", so that $X=K+\left[\left(K:{ }_{A} r^{n}\right) \cap r^{n} A\right]$. It will suffice to show that $X_{1}=\left(K:{ }_{A} r^{n}\right) \cap r^{n} A \leqq K$. Now $X_{1}:{ }_{A} r^{n} \leqq\left(K:{ }_{A} r^{n}\right):{ }_{A} r^{n}=K:{ }_{A} r^{2 n}=K:{ }_{A} r^{n}$. Hence $r^{n}\left(X_{1}:{ }_{A} r^{n}\right) \leqq r^{n}\left(K:{ }_{A} r^{n}\right) \leqq K$. But $r^{n}\left(X_{1}:{ }_{A} r^{n}\right)=X_{1} \cap r^{n} A=X_{1}$. Thus $X_{1} \leqq K$, as required. This completes the proof.

As an immediate consequence of Lemmas 7 and 8 we deduce 
Theorem 1. Let $A$ be a Noetherian object of the abelian category $(\mathscr{U}, \phi)$ over $R$. Then $A$ is fully structured.

We shall now begin an examination of the dual situation. To help with this we prove first of all the following result.

Lemma 9. Let $K$ and $\left\{N_{1}, N_{2}, \ldots, N_{m}\right\}$ be subobjects of $A$. Then

$$
K \equiv N_{1} \cap N_{2} \cap \ldots \cap N_{m}
$$

as subobjects of $A$ if und only if $(A / K)^{*} \equiv\left(A / N_{1}\right)^{*}+\left(A / N_{2}\right)^{*}+\ldots+\left(A / N_{m}\right)^{*}$ as subobjects of $A^{*}$. Dually, $K \equiv N_{1}+N_{2}+\ldots+N_{m}$ as subobjects of $A$ if and only if $(A / K)^{*} \equiv\left(A / N_{1}\right)^{*} \cap\left(A / N_{2}\right)^{*} \cap \ldots \cap\left(A / N_{m}\right)^{*}$ as subobjects of $A^{*}$.

Proof. These results follow readily from the equivalences

and

$$
\left(N_{1} \cap N_{2} \cap \ldots \cap N_{m}\right)^{*} \equiv A^{*} /\left\{\left(A / N_{1}\right)^{*}+\left(A / N_{2}\right)^{*}+\ldots+\left(A / N_{m}\right)^{*}\right\}
$$

$$
\left(N_{1}+N_{2}+\ldots+N_{m}\right)^{*} \equiv A^{*} /\left\{\left(A / N_{1}\right)^{*} \cap\left(A / N_{2}\right)^{*} \cap \ldots \cap\left(A / N_{m}\right)^{*}\right\} \text {. }
$$

Definition. The subobject $K$ of $A$ is said to have a coprimary decomposition in $A$ if the subobject $(A / K)^{*}$ of $A^{*}$ has a primary decomposition in $A^{*}$.

Lemma 10. The subobject $K$ of $A$ has a coprimary decomposition in $A$ if and only if $K$ is equivalent, as a subobject of $A$, to the sum of a finite number of coprimary subobjects of $A$.

Proof. $K$ has a coprimary decomposition in $A$ if and only if

$$
(A / K)^{*} \equiv N_{1}^{*} \cap \quad{ }_{2}^{*} \cap \ldots \cap N_{m}^{*},
$$

where $N_{i}^{*}$ is a $P_{i}$-primary subobject of $A^{*}(1 \leqq i \leqq m)$, which is so when and only when $K \equiv\left(A^{*} / N_{1}^{*}\right)^{*}+\left(A^{*} / N_{2}^{*}\right)^{*}+\ldots+\left(A^{*} / N_{m}^{*}\right)^{*}$. Furthermore, by definition, $\left(A^{*} / N_{i}^{*}\right)^{*}$ is a $P_{i}$-coprimary subobject of $A(1 \leqq i \leqq m)$.

Definition. The coprimary decomposition $K \equiv N_{1}+N_{2}+\ldots+N_{m}$ of $K$ in $A$ is said to be a coreduced coprimary decomposition of $K$ in $A$ if

$$
(A / K)^{*} \equiv\left(A / N_{1}\right)^{*} \cap\left(A / N_{2}\right)^{*} \cap \ldots \cap\left(A / N_{m}\right)^{*}
$$

is a reduced primary decomposition of $(A / K)^{*}$ in $A^{*}$.

Lemma 11. The coprimary decomposition $K \equiv N_{1}+N_{2}+\ldots+N_{m}$ of $K$ in $A$ is coreduced if and only if the following conditions are satisfied:

(a) $\mathscr{P}_{A, N_{i}}^{*} \neq \mathscr{P}_{A, N_{j}}^{*}(i \neq j)$

(b) no proper subset of $\left\{N_{1}, N_{2}, \ldots, N_{m}\right\}$ has sum equivalent to $K$.

Proof. This result follows readily from Lemma 9, the deduction made immediately before Lemma 3, and the definition of a reduced primary decomposition. 
Given a coprimary decomposition of a subobject $K$ of $A$, we obtain from it a primary decomposition of the subobject $(A / K)^{*}$ of $A^{*}$, and thence a reduced primary decomposition of $(A / K)^{*}$ in $A^{*}$. Finally, we may then obtain a coreduced coprimary decomposition of $K$ in $A$. Thus, given any coprimary decomposition we may refine it to yield a coreduced coprimary decomposition.

We now define an $\mathscr{U}$-object $A$ to be Artinian if and only if the dual object $A^{*}$ of the dual abelian category $\left(\mathscr{U}^{*}, \phi^{*}\right)$ over $R$ is Noetherian. Equivalently, we have the following

Definition. $A$ is said to be Artinian if, for every descending chain

$$
A_{1} \geqq A_{2} \geqq A_{3} \geqq \ldots
$$

of subobjects of $A$, there exists an integer $n$ such that $A_{n} \equiv A_{n+p}$ for $p=0,1,2, \ldots$.

Our next definition is of the dual property to that of being fully structured. An $\mathscr{U}$-object $A$ is said to be fully costructured if and only if the $\mathscr{U}^{*}$-object $A^{*}$ is fully structured. We may rephrase this definition in the following equivalent form.

Definition. An $\mathscr{U}$-object $A$ is fully costructured if and only if every subobject of $A$ has a coprimary decomposition in $A$.

The above definitions enable us to state the dual result to Theorem 1 , which is

Theorem 2. Let $A$ be an Artinian object of the abelian category $(\mathscr{U}, \phi)$ over $R$. Then $A$ is fully costructured.

\section{Uniqueness theorems}

Let $A$ be an object of the abelian category $(\mathscr{U}, \phi)$ over the ring $R$.

Lemma 12. Let $N$ be a P-primary subobject of $A$, and let $\Omega$ be a finitely generated ideal of $R$ such that $\Omega \notin P$. Then $N:_{A} \Omega=N$.

Proof. Suppose that $r \in \Omega \backslash P$. To obtain a contradiction, suppose that $N:{ }_{A} r \neq N$. The force of this assumption lies in the statement $N:{ }_{A} r \mid \underline{ }$. Now

$$
r\left(N:{ }_{A} r\right)=N \cap r A \leqq N,
$$

and so, since $N$ is a $P$-primary subobject of $A$, it follows that $r \in P$. This contradicts the choice of $r$, and so we deduce that $N:{ }_{A} r=N$, and hence that $N:_{A} \Omega=N$.

Proposition 1. Let $K$ be a subobject of $A$ and let $K \equiv N_{1} \cap N_{2} \cap \ldots \cap N_{m}$ be a primary decomposition of $K$ in $A$, where $N_{i}$ is a $P_{i}$-primary subobject of $A(1 \leqq i \leqq m)$. Then if $\Omega$ is a finitely generated ideal of $R$ such that

$$
\Omega \nsubseteq\left\{P_{1} \cup P_{2} \cup \ldots \cup P_{m}\right\}, K:{ }_{A} \Omega=K .
$$


Proof. Let $\Omega$ be generated by $\left\{\omega_{1}, \omega_{2}, \ldots, \omega_{n}\right\}$. Then

$$
\begin{aligned}
K::_{A} \Omega & =\bigcap_{i=1}^{n}\left(K:_{A} \omega_{i}\right) \\
& =\bigcap_{i=1}^{n}\left[\bigcap_{j=1}^{m} N_{j}\right]:_{A} \omega_{i} \\
& =\bigcap_{j=1}^{m}\left(\bigcap_{i=1}^{n}\left(N_{j}:_{A} \omega_{i}\right)\right) \\
& =\bigcap_{j=1}^{m}\left(N_{j}:_{A} \Omega\right) \\
& =\bigcap_{j=1}^{m} N_{j} \\
& =K,
\end{aligned}
$$

by Lemma 12 above.

Lemma 13. Let $N$ be a P-primary subobject of $A$, and let $\Omega$ be a finitely generated ideal of $R$ such that $N:_{A} \Omega=N$. Then $\Omega \ddagger P$.

Proof. Suppose $\left\{\omega_{1}, \omega_{2}, \ldots, \omega_{n}\right\}$ generates the ideal $\Omega$. Assume, to obtain a contradiction, that $\Omega \subseteq P$. Since $\Omega \subseteq P$, there exist positive integers

$$
m_{1}, m_{2}, \ldots, m_{n}
$$

such that $\omega_{i}^{m_{i}} A \leqq N(1 \leqq i \leqq n)$. Setting $m=m_{1}+m_{2}+\ldots+m_{n}$ we have $\Omega^{m} A \leqq N$. Let $k$ be the smallest positive integer such that $\Omega^{k} A \leqq N$. Then $\Omega^{k-1} A$

$$
\omega_{i}\left(\Omega^{k-1} A\right) \leqq \Omega\left(\Omega^{k-1} A\right)=\Omega^{k} A \leqq N .
$$

Hence $\Omega^{k-1} A \leqq \bigcap_{i=1}^{n}\left(N:_{A} \omega_{i}\right)=N:_{A} \Omega$. Thus we have $\Omega^{k-1} A \leqq N:_{A} \Omega$, and $\Omega^{k-1} A \pm N$, so that $\left(N:_{A} \Omega\right) \neq N$, from which contradiction it follows that $\Omega \nsubseteq P$.

Proposition 2. Let the subobject $K$ of $A$ have a reduced primary decomposition $K \equiv N_{1} \cap N_{2} \cap \ldots \cap N_{m}$ in $A$, where $N_{i}$ is a $P_{i}$-primary subobject of $A(1 \leqq i \leqq m)$. Let $\Omega$ be a finitely generated ideal of $R$ such that $\left(K:_{A} \Omega\right)=K$. Then

$$
\Omega \nsubseteq\left\{P_{1} \cup P_{2} \cup \ldots \cup P_{m}\right\} .
$$

Proof. We may assume that $K$ is a proper subobject of $A$. By induction we know that $K:{ }_{A} \Omega^{n}=K$ for every integer $n$, since $K:_{A} \Omega=K$.

Let $I$ denote the subset of $\{1,2, \ldots, m\}$ consisting of those integers $i$ for which $\Omega \subseteq P_{i}$. Suppose that $I$ is non-empty. We shall assume that $I=\{1,2, \ldots, l\}$ and set $J=\{l+1, l+2, \ldots, m\}$. Now by Lemma 13 there exist positive integers 
$n_{1}, n_{2}, \ldots, n_{l}$ such that $\Omega^{n_{i}} A \leqq N_{i}(1 \leqq i \leqq l)$. Hence $\left(N_{i}: \Omega^{n^{n}}\right) \leqq A$ for $1 \leqq i \leqq l$. Setting $n=\max \left\{n_{1}, n_{2}, \ldots, n_{l}\right\}$, we see that $N_{i}: A \Omega^{n}=A(1 \leqq i \leqq l)$. Now

$$
\begin{aligned}
K & =K:_{A} \Omega^{n} \\
& =\left[\bigcap_{i=1}^{m} N_{i}\right]: \Omega_{A} \Omega^{n} \\
& =\bigcap_{i=1}^{m}\left(N_{i}:_{A} \Omega^{n}\right) \\
& =A \cap \bigcap_{i=1+1}^{m}\left(N_{i}: \Omega_{A} \Omega^{n}\right) \\
& =\bigcap_{i=1}^{m}\left(N_{i}:_{A} \Omega^{n}\right) .
\end{aligned}
$$

But $\Omega \nsubseteq P_{j}(l+1 \leqq j \leqq m)$, and each $P_{j}$ is a prime ideal, so that it follows that $\Omega^{n} \ddagger P_{j}(l+1 \leqq j \leqq m)$. Hence, by Lemma $12, N_{j}: \Omega^{n}=N_{j}(l+1 \leqq j \leqq m)$. Therefore $K \equiv N_{t+1} \cap N_{t+2} \cap \ldots \cap N_{m}$, contradicting the fact that

$$
K \equiv N_{1} \cap N_{2} \cap \ldots \cap N_{m}
$$

was a reduced primary decomposition of $K$ in $A$. Thus $I$ must be empty, as required.

We shall now assume that $K$ is a subobject of $A$, and that $K$ has two reduced primary decompositions in $A$,

and

$$
K \equiv N_{1} \cap N_{2} \cap \ldots \cap N_{l}
$$

$$
K \equiv N_{1}^{\prime} \cap N_{2}^{\prime} \cap \ldots \cap N_{m}^{\prime},
$$

where $N_{i}$ is a $P_{i}$-primary subobject of $A(1 \leqq i \leqq l)$ and $N_{j}^{\prime}$ is a $P_{j}^{\prime}$-primary subobject of $A(1 \leqq j \leqq m)$. Let $r \in R \backslash\left\{P_{1} \cup P_{2} \cup \ldots \cup P_{l}\right\}$. By Proposition 1 we know that $K:_{A} r=K$, so that from Proposition 2 we have

$$
r \in R \backslash\left\{P_{1}^{\prime} \cup P_{2}^{\prime} \cup \ldots \cup P_{m}^{\prime}\right\} .
$$

Hence it follows that $\left\{P_{1}^{\prime} \cup P_{2}^{\prime} \cup \ldots \cup P_{m}^{\prime}\right\} \subseteq\left\{P_{1} \cup P_{2} \cup \ldots \cup P_{l}\right\}$. The opposite inclusion follows similarly so that we have $\left\{P_{1} \cup P_{2} \cup \ldots \cup P_{l}\right\}=\left\{P_{1}^{\prime} \cup P_{2}^{\prime} \cup \ldots \cup P_{m}^{\prime}\right\}$. Therefore we make the following

Definition. Let the subobject $K$ of $A$ have a reduced primary decomposition $K \equiv N_{1} \cap N_{2} \cap \ldots \cap N_{m}$ in $A$, where $N_{i}$ is a $P_{i}$-primary subobject of $A(1 \leqq i \leqq m)$. Then the prime set of $K$ in $A$ is defined to be the set-theoretic union of the prime ideals $P_{1}, P_{2}, \ldots, P_{m}$.

From Propositions 1 and 2 we deduce

Lemma 14. Suppose that the subobject $K$ of $A$ has a reduced primary decomposition in $A$. Then the prime set of $K$ in $A$ is $\left\{r \in R:\left(K:{ }_{A} r\right) \neq K\right\}$. 
We again suppose that

and

$$
K \equiv N_{1} \cap N_{2} \cap \ldots \cap N_{l}
$$

$$
K \equiv N_{1}^{\prime} \cap N_{2}^{\prime} \cap \ldots \cap N_{m}^{\prime}
$$

are reduced primary decompositions of $K$ in $A$, where $N_{i}$ is a $P_{i}$-primary subobject of $A(1 \leqq i \leqq l)$ and $N_{j}^{\prime}$ is a $P_{j}^{\prime}$-primary subobject of $A(1 \leqq j \leqq m)$. Suppose that $\left\{P_{1}, P_{2}, \ldots, P_{q}\right\}$ and $\left\{P_{1}^{\prime}, P_{2}^{\prime}, \ldots, P_{r}^{\prime}\right\}$ are the maximal members of $\left\{P_{1}, P_{2}, \ldots, P_{l}\right\}$ and $\left\{P_{1}^{\prime}, P_{2}^{\prime}, \ldots, P_{m}^{\prime}\right\}$ respectively. Now

$$
P_{1} \subseteq\left\{P_{1} \cup P_{2} \cup \ldots \cup P_{l}\right\}=\left\{P_{1}^{\prime} \cup P_{2}^{\prime} \cup \ldots \cup P_{m}^{\prime}\right\}
$$

so that there exists $1 \leqq j \leqq m$ such that $P_{1} \subseteq P_{j}^{\prime}$. We may choose $j$ so that $P_{j}^{\prime}$ is maximal in $\left\{P_{1}^{\prime}, P_{2}^{\prime}, \ldots, P_{m}^{\prime}\right\}$. Similarly,

$$
P_{j}^{\prime} \subseteq\left\{P_{1} \cup P_{2} \cup \ldots \cup P_{l}\right\},
$$

so that there exists $1 \leqq i \leqq l$ such that $P_{j}^{\prime} \subseteq P_{i}$. Since $P_{1}$ is a maximal member of $\left\{P_{1}, P_{2}, \ldots, P_{1}\right\}$, and $P_{1} \subseteq P_{j}^{\prime} \subseteq P_{i}$, it follows that $P_{1}=P_{j}^{\prime}$.

Repeating the above argument a suitable number of times we deduce that the two sets of prime ideals $\left\{P_{1}, P_{2}, \ldots, P_{q}\right\}$ and $\left\{P_{1}^{\prime}, P_{2}^{\prime}, \ldots, P_{r}^{\prime}\right\}$ must be the same.

This result may however be generalised in the following manner.

Theorem 3. Let the subobject $K$ of $A$ have two reduced primary decompositions

and

$$
K \equiv N_{1} \cap N_{2} \cap \ldots \cap N_{l}
$$

$$
K \equiv N_{1}^{\prime} \cap N_{2}^{\prime} \cap \ldots \cap N_{m}^{\prime}
$$

in $A$, where $\mathscr{P}_{A, N_{i}}=P_{i}(1 \leqq i \leqq l)$ and $\mathscr{P}_{A, N_{j}^{\prime}}=P_{j}^{\prime}(1 \leqq j \leqq m)$. Then $l=m$ and there is a permutation $\sigma$ of $\{1,2, \ldots, l\}$ such that $P_{i}=P_{\sigma(i)}^{\prime}(1 \leqq i \leqq l)$.

Proof. The proof is by induction on the integer $n=\min (l, m)$. If $n=0$ then $K=A$, and the result is therefore trivially true in this case.

Assume now that $n$ is a positive integer and that the required result holds for any subobject of $A$ having at least one reduced primary decomposition as the intersection of at most $n-1$ primary subobjects of $A$.

Consider the set $\left\{P_{1}, P_{2}, \ldots, P_{l}, P_{1}^{\prime}, P_{2}^{\prime}, \ldots, P_{m}^{\prime}\right\}$ of prime ideals. Let $P$ be a maximal member of this set. $P$ must belong to both $\left\{P_{1}, P_{2}, \ldots, P_{t}\right\}$ and to $\left\{\boldsymbol{P}_{1}^{\prime}, \boldsymbol{P}_{2}^{\prime}, \ldots, \boldsymbol{P}_{m}^{\prime}\right\}$. Assume without loss of generality that $N_{1}$ and $N_{1}^{\prime}$ are $P$-primary subobjects of $A$. We may choose $r \in P$ such that

$$
r \notin P_{2}, r \notin P_{3}, \ldots, r \notin P_{l}
$$

and such that $r \notin P_{2}^{\prime}, r \notin P_{3}^{\prime}, \ldots, r \notin P_{m}^{\prime}$. Now there exist positive integers $v_{1}, v_{2}$ such that $r^{v_{1}} A \leqq N_{1}$ and $r^{v_{2}} A \leqq N_{1}^{\prime}$. Setting $v=\max \left\{v_{1}, v_{2}\right\}$, we have

$$
N_{1}: r^{v}=A
$$


and

$$
N_{1}^{\prime}:{ }_{A} r^{\nu}=A .
$$

But $r^{\nu} \notin P_{j}$ for any $j$ satisfying $2 \leqq j \leqq l$, and so

Hence

$$
N_{j}: r^{v}=N_{j} \quad(2 \leqq j \leqq l) .
$$

Similarly

$$
K:{ }_{A} r^{v}=\bigcap_{j=1}^{l}\left(N_{j}: r_{A} r^{v}\right)=N_{2} \cap N_{3} \cap \ldots \cap N_{i} .
$$

$$
K:{ }_{A} r^{\nu}=N_{2}^{\prime} \cap N_{3}^{\prime} \cap \ldots \cap N_{m}^{\prime} .
$$

But each of these primary decompositions of $K:{ }_{A} r^{\nu}$ in $A$ is reduced. By the inductive hypothesis, it follows that $l-1=m-1$, so that $l=m$, and also there is a permutation $\sigma$ of $\{2,3, \ldots, l\}$ such that $P_{i}=P_{\sigma(i)}^{\prime}(2 \leqq i \leqq l)$. Setting $\sigma(1)=1$, we obtain the required permutation of $\{1,2, \ldots, l\}$. This completes the proof of the theorem.

Definition. Let $K$ be a subobject of $A$ having a reduced primary decomposition

$$
K \equiv N_{1} \cap N_{2} \cap \ldots \cap N_{m}
$$

in $A$. Then the ideals $\mathscr{P}_{A, N_{1}}, \mathscr{P}_{A, N_{2}}, \ldots, \mathscr{P}_{A, N_{m}}$ will be called the prime ideals belonging to $K$ in $A$.

From Theorem 3 the concept just introduced is well-defined.

We turn now to the dual theory. From Lemma 1 and the equivalences stated in the proof of Lemma 9, we may readily deduce that if $K$ is a subobject of the $\mathscr{U}$-object $A$, and $\Omega$ is a finitely generated ideal of $R$, then the subobjects $(A / \Omega K)^{*}$ and $(A / K)^{*}:_{A^{*}} \Omega$ of $A^{*}$ are equivalent. This enables us to deduce Propositions 3 and 4, which are the duals of Propositions 1 and 2.

Proposition 3. Let $K$ be a subobject of $A$, having $K \equiv N_{1}+N_{2}+\ldots+N_{m}$ as a coprimary decomposition in $A$, where $N_{i}$ is a $P_{i}$-coprimary subobject of $A(1 \leqq i \leqq m)$. Then, if $\Omega$ is a finitely generated ideal of $R$ such that

$$
\Omega \nsubseteq\left\{P_{1} \cup P_{2} \cup \ldots \cup P_{m}\right\}, \quad \Omega K=K .
$$

Proof. By Lemma 9, $(A / K)^{*} \equiv\left(A / N_{1}\right)^{*} \cap\left(A / N_{2}\right)^{*} \cap \ldots \cap\left(A / N_{m}\right)^{*}$, where $\left(A / N_{i}\right)^{*}$ is a $P_{i}$-primary subobject of $A^{*}(1 \leqq i \leqq m)$, since

$$
\mathscr{P}_{A^{*},\left(A / N_{i}\right)^{*}}=\mathscr{P}_{A, N_{i}}^{*}=P_{i} \text {. }
$$

Since $\Omega \nsubseteq\left\{P_{1} \cup P_{2} \cup \ldots \cup P_{m}\right\}$, we deduce from Proposition 1 that

$$
(A / K)^{*}:_{A^{*}} \Omega=(A / K)^{*},
$$

and so $(A / \Omega K)^{*}=(A / K)^{*}$, which is equivalent to $\Omega K=K$.

Proposition 4. Let $K$ be a subobject of $A$, having a coreduced coprimary decomposition $K \equiv N_{1}+N_{2}+\ldots+N_{m}$ in $A$, where $N_{i}$ is a $P_{i}$-coprimary subobject 
of $A(1 \leqq i \leqq m)$. Let $\Omega$ be a finitely generated ideal of $R$ such that $\Omega K=K$. Then $\Omega \neq\left\{P_{1} \cup P_{2} \cup \ldots \cup P_{m}\right\}$.

Proof. This is deduced from Proposition 2 in a similar manner to that in which Proposition 3 resulted from Proposition 1.

Suppose now that $K$ is a subobject of $A$ having a coreduced coprimary decomposition in $A$. Then we define the coprime set of $K$ in $A$ to be the prime set of $(A / K)^{*}$ in $A^{*}$. By duality we are able to express this in a different form. This is the aim of

Lemma 15. Let the subobject $K$ of $A$ have a coreduced coprimary decomposition $K \equiv N_{1}+N_{2}+\ldots+N_{m}$ in $A$, where $N_{i}$ is a $P_{i}$-coprimary subobject of $A(1 \leqq i \leqq m)$. Then the coprime set of $K$ in $A$ is the union of the prime ideals $\left\{P_{1}, P_{2}, \ldots, P_{m}\right\}$. It is also characterised as $\{r \in R: r K \neq K\}$, and is therefore independent of the particular coprimary decomposition of $K$ in $A$ under consideration.

We shall now conclude this section with the dual of Theorem 3 , and the definition of the resulting dual concept.

Theorem 4. Let $K$ be a subobject of $A$ having two coreduced coprimary decompositions

and

$$
K \equiv N_{1}+N_{2}+\ldots+N_{l}
$$

$$
K \equiv N_{1}^{\prime}+N_{2}^{\prime}+\ldots+N_{m}^{\prime}
$$

in $A$, where $\mathscr{P}_{A, N_{i}}^{*}=P_{i}(1 \leqq i \leqq l)$ and $\mathscr{P}_{A, N_{j}^{*}}^{*}=P_{j}^{\prime}(1 \leqq j \leqq m)$. Then $l=m$ and there is a permutation $\sigma$ of $\{1,2, \ldots, l\}$ such that $P_{i}=P_{\sigma(i)}^{\prime}(1 \leqq i \leqq l)$.

Definition. Let the subobject $K$ of $A$ have a coreduced coprimary decomposition $K \equiv N_{1}+N_{2}+\ldots+N_{m}$ in $A$. Then the ideals $\mathscr{P}_{A, N_{1}}^{*}, \mathscr{P}_{A, N_{2}}^{*}, \ldots, \mathscr{P}_{A, N_{m}}^{*}$ are called the prime ideals cobelonging to $K$ in $A$.

\section{An application}

We now make an application of our earlier results to obtain a well-known result from commutative algebra, together with its dual.

Theorem 5. Let the subobject $K$ of $A$ have a primary decomposition in $A$, and let $\Omega$ be a finitely generated ideal of the ring $R$. Then $K:_{A} \Omega=K$ when and only when there exists $\omega \in \Omega$ such that $K:_{A} \omega=K$.

Proof. Let $K \equiv N_{1} \cap N_{2} \cap \ldots \cap N_{m}$ be a reduced primary decomposition of $K$ in $A$, where $N_{i}$ is a $P_{i}$-primary subobject of $A$. From Propositions 1 and 2 , $K:{ }_{A} \Omega=K$ if and only if $\Omega \nsubseteq P_{1}, \Omega \nsubseteq P_{2}, \ldots, \Omega \nsubseteq P_{m}$. But by induction on $m$, this is so when and only when we can choose $\omega \in \Omega$ such that

$$
\omega \notin P_{1}, \omega \notin P_{2}, \ldots, \omega \notin P_{m} \text {. }
$$

Again using Propositions 1 and 2 , this is so if and only if $K:_{A} \omega=K$. 
Theorem 6. Let the subobject $K$ of $A$ have a coprimary decomposition in $A$ and let $\Omega$ be a finitely generated ideal of $R$. Then $\Omega K=K$ if and only if there exists $\omega \in \Omega$ such that $\omega K=K$.

Proof. This follows from Theorem 5 and the equivalence of the subobjects $(A / \Omega K)^{*}$ and $(A / K)^{*}: A^{*} \Omega$ of $A^{*}$.

\section{An example}

In this section we give a simple example of the use of the ideas that we have encountered.

Let $p_{1}, p_{2}, \ldots, p_{k}$ be distinct prime integers, and let $n_{1}, n_{2}, \ldots, n_{k}$ be non-negative integers. We shall let $A$ denote the additive group of integers modulo $p_{1}^{n_{1}} p_{2}^{n_{2}} \ldots p_{k}^{n_{k}}$. $A$ can then be regarded as a Z-module in the usual manner, where $\mathbf{Z}$ denotes the ring of integers. Every submodule of $A$ may be singly generated, and we shall use $(d)$ to denote the submodule of $A$ which is generated by the integer $d$.

The primary submodules of the Z-module $A$ are those generated by an element of $A$ of the form $p_{1}^{\mu_{1}} p_{2}^{\mu_{2}} \ldots p_{k}^{\mu_{k}}$, where all but one of the $\mu_{i}$ 's is zero, and the single non-zero $\mu_{i}$ satisfies $1 \leqq \mu_{i} \leqq n_{i}$.

The coprimary submodules of $A$ are those generated by elements of $A$ of the form $p_{1}^{\mu_{1}} p_{2}^{\mu_{2}} \ldots p_{k}^{\mu_{k}}$, where this time all but one of the $\mu_{i}$ 's equals the corresponding $n_{i}$, and the single exception satisfies $0 \leqq \mu_{i}<n_{i}$.

$A$, being a finite Z-module, is both Noetherian and Artinian, and so each submodule of $A$ has a primary and a coprimary decomposition in $A$. The submodule of $A$ generated by $p_{1}^{m_{1}} p_{2}^{m_{2}} \ldots p_{k}^{m_{k}}$, where $0 \leqq m_{i} \leqq n_{i}(1 \leqq i \leqq k)$, has a primary decomposition

$$
\left(p_{1}^{m_{1}} p_{2}^{m_{2}} \ldots p_{k}^{m_{k}}\right)=\bigcap_{\substack{i=1 \\ m_{i} \neq 0}}^{k}\left(p_{i}^{m_{i}}\right)
$$

in $A$. Again, it is easily seen that

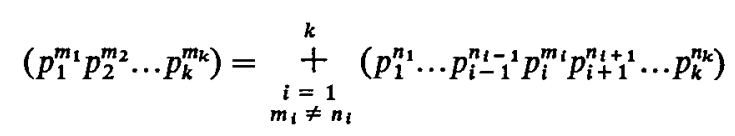

is a coprimary decomposition of $\left(p_{1}^{m_{1}} p_{2}^{m_{2}} \ldots p_{k}^{m_{k}}\right)$ in $A$.

\section{REFERENCES}

(1) P. Gabriel, Des Catégories Abéliennes, Bull. Soc. Math. France, 90 (1962), 323-448.

(2) E. MatuIs, Modules with Descending Chain Condition, Trans. Amer. Math. Soc. 97 (1960), 495-508.

$$
\text { E.M.S.-18/4-B }
$$


(3) D. J. MOORE, 'Some Remarks on $R$-Sequences and $R$-Cosequences, $J$. London Math. Soc. (to appear).

(4) D. G. NoRthCotT, Lessons on Rings, Modules and Multiplicities (Cambridge University Press, 1968).

UNIVERSITY OF GLASGOW

SCOTLAND 